\title{
Possibility to use of the $\mathrm{Fe}_{3} \mathrm{O}_{4} / \mathrm{Ta}_{2} \mathrm{O}_{5}$ core-shell nanoparticles in radiotherapy
}

\author{
Kseniya Lukyanenko ${ }^{1, *}$, Leonid Afremov ${ }^{1}$, Vladimir Apanasevich ${ }^{2}$, Mariya Shmykova ${ }^{1}$, Mikhail Medkov ${ }^{3}$ Pavel \\ Lukyanov $^{4}$, Olga Tarakova ${ }^{1}$, Vladimir Kustov ${ }^{5}$ \\ ${ }^{1}$ Far Eastern Federal University, 690950, 8 Sukhanova St., Russia \\ ${ }^{2}$ Pacific State Medical University, 690002, 2 Ostryakova Avenue, Russia \\ ${ }^{3}$ Institute of Chemistry FEB RAS, 690022, 159, Prospekt 100-let Vladivostoku, Russia \\ ${ }^{4}$ G.B. Elyakov Pacific Institute of Bioorganic Chemistry FEB RAS, 690022,159 Prospect 100 let Vladivostoku, Russia \\ ${ }^{5}$ Vladivostok's Branch of the Customs Academy, 690034, 16v Strelkovaya st., Russia
}

\begin{abstract}
The study was carried out of the possibility of using magnetic core-shell nanoparticles $\mathrm{Fe} 3 \mathrm{O} 4 / \mathrm{Ta} 2 \mathrm{O} 5$ as a radio-modifier. It is investigated the influence of the inhomogeneous magnetic field on the distribution of the nanoparticles in the region of its maximum inhomogeneity. The increase of the coreshell nanoparticles' concentration leads to the increase of the number of $511 \mathrm{keV}$ gamma-quanta. The absorption of gamma-quanta with lower energy $(20-200 \mathrm{keV})$ increases with increase in concentration of nanoparticles.
\end{abstract}

\section{Introduction}

A development of the biomedical technologies including such as hyperthermia [1-4], targeted drug delivery [5-11] as well as a creation of biosensors and biochips [12] resulted in the heavy growth in the experimental investigations of the magnetic properties of the systems of different nanoparticles and nanofilms/nanotape. The possibility to manipulate living cells anchored with NPs via a magnetic field, ferromagnetic NPs affect hemopoietic /stromal cells and promote cellular adhesion and formation of cell-to-cell contacts along the magnetic field lines, NPs in regenerative medicine, thermal ablation, etc. are describe in $[13,14]$.

Over the last years, the interest has increased in the study of magnetic nanoparticles for purposes of their use in the cancer diagnostics and treatment. The unique properties of the nanoparticles (NPs), ability to control their geometric (sizes, shapes) and surface characteristics determining the hydrophility and hydrophobicity of the matter as well as possibility to inject the nanoparticles to the biological media in a number of different ways make them perspective in many aspects of oncology.

Magnetic core-shell nanoparticles are important for applications because they allow to control them via an external magnetic field. Direct control of the motion and distribution of magnetic nanoparticles forms at the basis of their using for targeted drug delivery or for the delivery of magnetic nanoparticles to cancer tumors [7, 10] for the purpose of the subsequent hyperthermia process or to enhance the effect of radiation therapy due to scattering radiation by injected particles.

Various published data referred to as iron oxide toxicity [15] and its biocompatibility [16]. At the present time, the studies related to obtaining the nontoxic magnetic nanoparticles based on iron oxides covered, for example, with the tantalum oxide and designed to biomedical applications become more and more actual [17]. In addition, tantalum oxide nanoparticles have a long circulation period in the bloodstream and they are usually easily biodegradable and have low toxicity [18].

It should be noted that the core-shell $\mathrm{Fe}_{3} \mathrm{O}_{4} / \mathrm{Ta}_{2} \mathrm{O}_{5}$ nanoparticles can be used to construct magnetically controlled non-toxic X-ray contract drugs [19].

The objective of this paper is to study the possibility of using magnetic core-shell nanoparticles of $\mathrm{Fe}_{3} \mathrm{O}_{4} / \mathrm{Ta}_{2} \mathrm{O}_{5}$ to enhance the effectiveness of radiation therapy.

\section{Synthesis of nanoparticles of $\mathrm{Fe}_{3} \mathrm{O}_{4} / \mathrm{Ta}_{2} \mathrm{O}_{5}$ and their properties}

For achievement of the above-referenced objective, we have synthesized the core/shell nanoparticles the cores of which consist of the iron oxide while envelope of the tantalum oxide.

To achieve the above goal, we synthesized $\mathrm{Fe} 3 \mathrm{O} 4 / \mathrm{Ta} 2 \mathrm{O} 5$ nanoparticles. An aqueous fluoride solution of tantalum was used as a precursor, and sodium oleate as a stabilizer of nanoparticles, in contrast to the widely used method of obtaining a core-shell structure [15]. To a solution containing $50 \mathrm{ml}$ of ammonia was added $30 \mathrm{ml}$ solution containing $0.3 \mathrm{~g}$ of tantalum in hydrofluoric acid with stirring. Further the pulp was allowed to settle, decanted some of the solution over the precipitate, and the remaining pulp was filtered. The precipitate was washed on the filter twice, after which the precipitate was pelletized in $100 \mathrm{ml}$ of water. To the

\footnotetext{
* Corresponding author: ks.lukyanenko@gmail.com
} 
received of the mixture was added successively $100 \mathrm{ml}$ of a solution containing $0.5 \mathrm{~g}$ of $\mathrm{FeSO}_{4} .7 \mathrm{H}_{2} \mathrm{O}$ and $0.7 \mathrm{~g}$ of $\mathrm{Fe}_{2}\left(\mathrm{SO}_{4}\right)_{3}, 0.2 \mathrm{ml}$ of a $5 \%$ solution of sodium oleate and $50 \mathrm{ml}$ of concentrated ammonia solution with stirring. After that, $15 \mathrm{ml}$ of a solution of tantalum in hydrofluoric acid, $50 \mathrm{ml}$ of ammonia and $0.2 \mathrm{ml}$ of $5 \%$ solution of sodium oleate. The $\mathrm{pH}$ value in the stages of tantalum deposition and the iron should be not less than 10. Then the pulp was allowed to settle, poured decanting part of the solution over the precipitate, and the remaining pulp was filtered. The precipitate on the filter is washed twice, after which it is pulped precipitate in $100 \mathrm{ml}$ of water. The resulting pulp was ultrasonically dispersed. The result was a magnetic fluid that was magnetically radiopaque agent. Advantages of the proposed method, we obtained as described in [20], are associated with fewer stages of the process, which significantly reduces the synthesis time of nanoparticles.

The geometric characteristics of nanoparticles and their size distribution were determined using the atomicforce microscope. The atomic-force microscopy allowed us to construct the size distribution (Fig.1). It is described by close to normal law of distribution with mean value $d=44 \mathrm{~nm}$ and variance $\sigma=3 \mathrm{~nm}$.

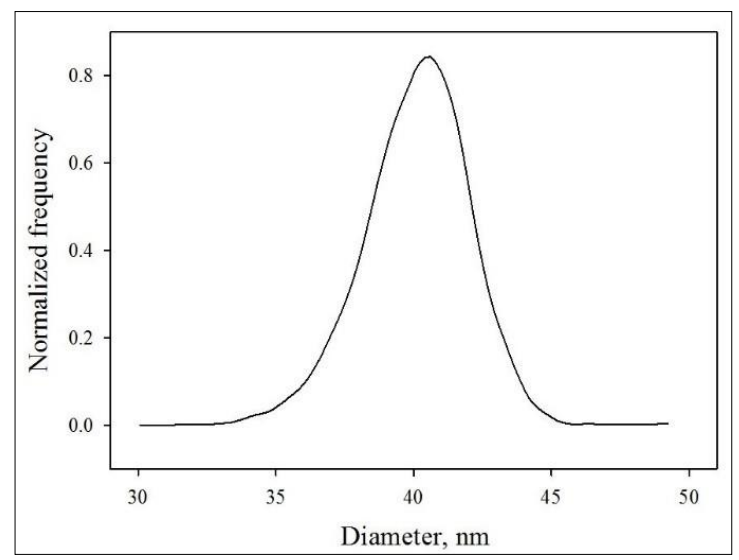

Fig. 1. The size distribution of $\mathrm{Fe}_{3} \mathrm{O}_{4} / \mathrm{Ta}_{2} \mathrm{O}_{5}$ core-shell magnetic NPs with the use of AFM.

\section{Nanoparticles distribution in the magnetic field}

A modeling of the $\mathrm{Fe}_{3} \mathrm{O}_{4} / \mathrm{Ta}_{2} \mathrm{O}_{5}$ nanoparticles distribution in the magnetic field was performed in the sodium oleate solution. For this, the cuvette with the dissolved $\mathrm{Fe}_{3} \mathrm{O}_{4} / \mathrm{Ta}_{2} \mathrm{O}_{5}$ nanoparticles was placed into the inhomogeneous magnetic field $H$ established by the coil with the pointed ferromagnetic core. Recording the image brightness was performed using the digital microscope and, afterwards, the mathematical processing of images obtained was accomplished.

Recording the image brightness was carried out on two solutions the concentrations of nanoparticles $\Delta \mathrm{n}_{\mathrm{n}}$ in which differ twice. We assume that the image brightness is proportional to relative concentration of nanoparticles $\Delta n n_{0}$ in the solution. In Fig. 2, the image brightness of solution in different fields is shown.

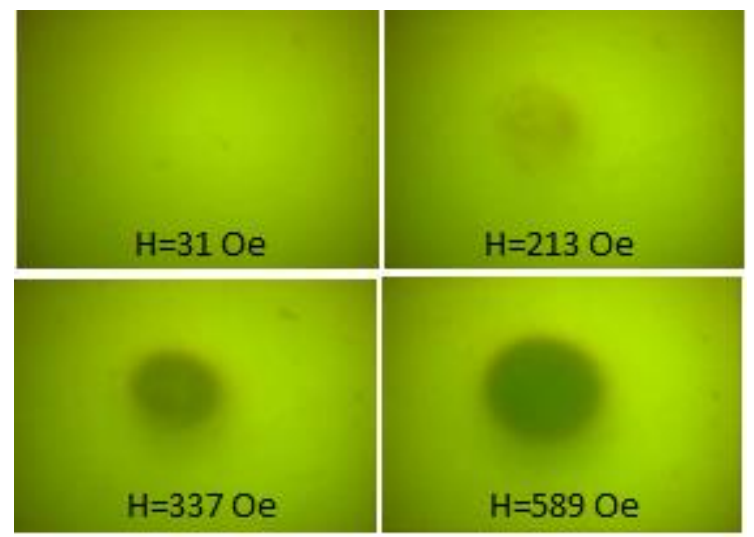

Fig. 2. Light intensity of the solution in different fields $H$.

The dependence of the distribution of relative nanoparticles concentration in the area of solution near the spot center on the magnetic field and initial concentration of particles is presented in Fig. 3. The effective spot size $R_{\text {eff }}$ increases with magnetic field's increasing and decreases with decreasing of the nanoparticle concentration as expected.
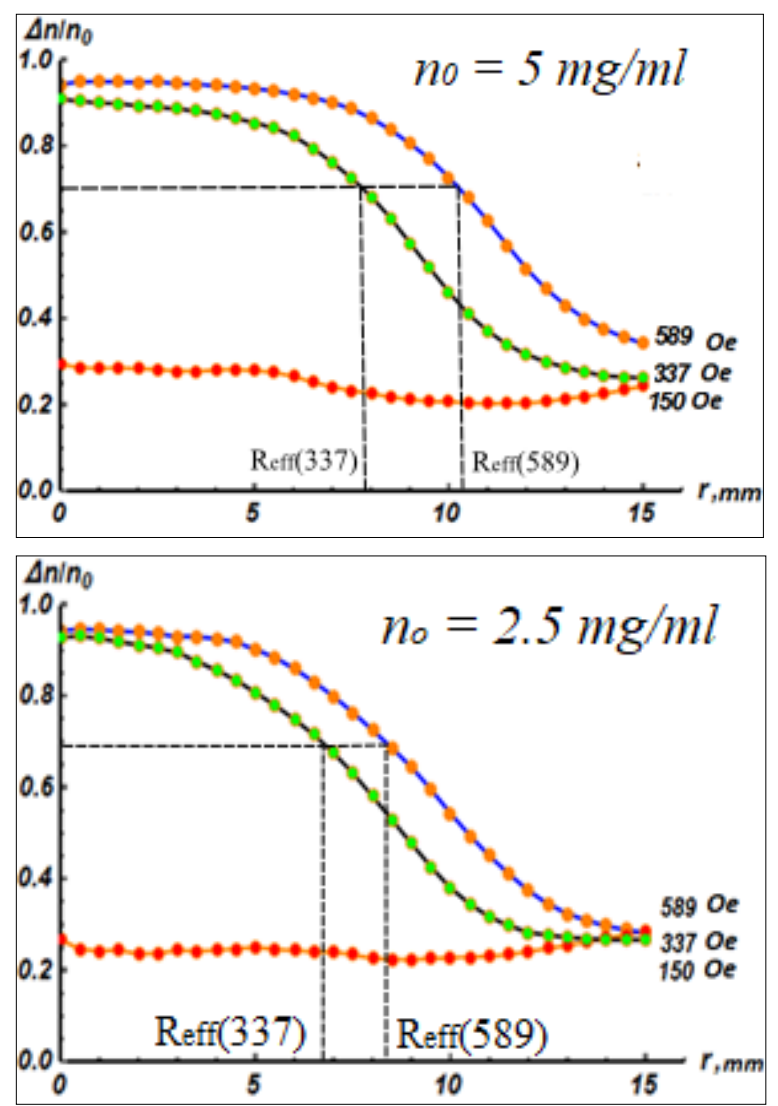

Fig. 3. Distribution of the relative concentration of the NPs $\Delta n n_{0}$ on the magnetic field $H(\mathrm{Oe})$ and solution density $n_{0} . r-$ distance between the cloud center and measuring range.

From Fig. 4, it is evident that the relative concentration of nanoparticles at the spot center increases with increase in the magnetic field strength and reaches the saturation at its particular value. The initial concentration of nanoparticles $n_{0}$ in the solution determines the value of the magnetic field at which the curve of relative concentration dependence attains the 
saturation (Fig. 4): the lesser magnetic field corresponds to the larger initial concentration.

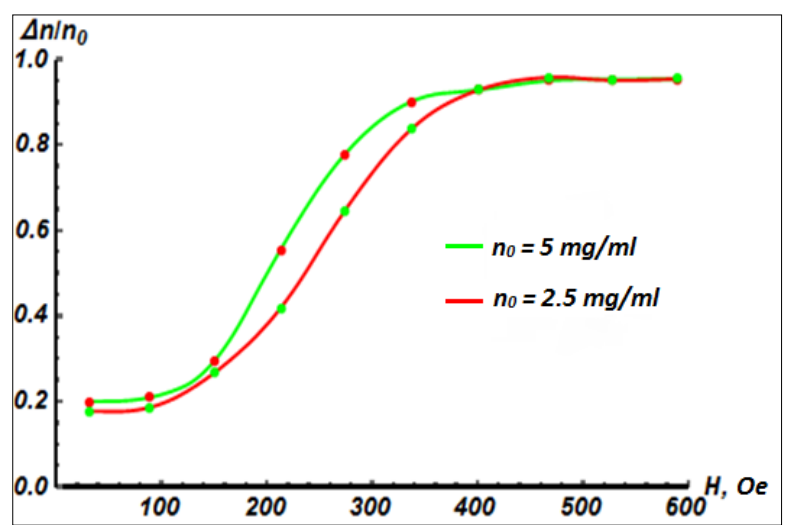

Fig. 4. Dependence of the relative concentration $\Delta n n_{0}$ of the NPs in cloud center on magnetic field $H$

Therefore, for the nanoparticles under consideration, the major values of the magnetic field needed for their maximal concentration at the fixed point.

\section{Energy spectrum of gamma-ray emission absorption}

The major objective of the modern therapy of the oncology diseases is to defeat the tumor cells with minimum damage to the healthy tissues. As it's known, the maximum biological effect is exercised by the kilovolt radiation (20-200 keV) [21].

One expects that the introduction of nanoparticles containing tantalum into the area of malignant tumor should result in increase of the number of the low-energy quanta which will contribute to the apoptosis of the tumor cells. In order to test this hypothesis, we conducted the experimental study of the influence of the $\mathrm{Fe}_{3} \mathrm{O}_{4} / \mathrm{Ta}_{2} \mathrm{O}_{5}$ nanoparticle concentration on the energy spectrum of gamma-ray radiation absorption. In the experiment in vitro, we used the gamma-ray spectrometer with the semiconductor detector GEM$15 \mathrm{P} 4$ and two gamma radiation sources ${ }^{60} \mathrm{Co}$ (sample spectrometry gamma source type) with the aggregate activity of about $2 \times 10^{5} \mathrm{~Bq}$. The concentration of nanoparticles in the specified area was changed by use of magnetic field: before each radiation, the nanoparticles were subjected to impact of different magnetic fields, at that, the delay time for each measurement was 5 minutes. The curves of dependence of the gamma-quanta number $\Delta n=n(H)-n(H=0)$ on their number in the field $H=0$ are shown in Fig. 5 .

The increase in the magnetic field force and, thereby, concentration of nanoparticles $\mathrm{Fe}_{3} \mathrm{O}_{4} / \mathrm{Ta}_{2} \mathrm{O}_{5}$, result in growth in the number of quanta in the range of $511 \mathrm{keV}$. Most likely, such behavior of the absorption curve is related to the creation of electron-positron pairs [22]. As the substantiation of this statement, the fact that the energy of gamma-quant higher than $2 \mathrm{mc}^{2}=1022 \mathrm{keV}$ is needed for the creation of electron-positron pair can serve. While the energy of the ${ }^{60} \mathrm{Co}$ gamma-ray is slightly higher and is equal to $1.25 \mathrm{MeV}$. The electron and positron formed as a result of the interaction between the gamma-ray and substance are absorbed in the radiated tissues, in what connection, the positron annihilates, producing at this two secondary photons with energy of $511 \mathrm{keV}$ each. The secondary gammaquanta with lower, than the primary radiation, energy have the higher potential of interaction with the biological environment.

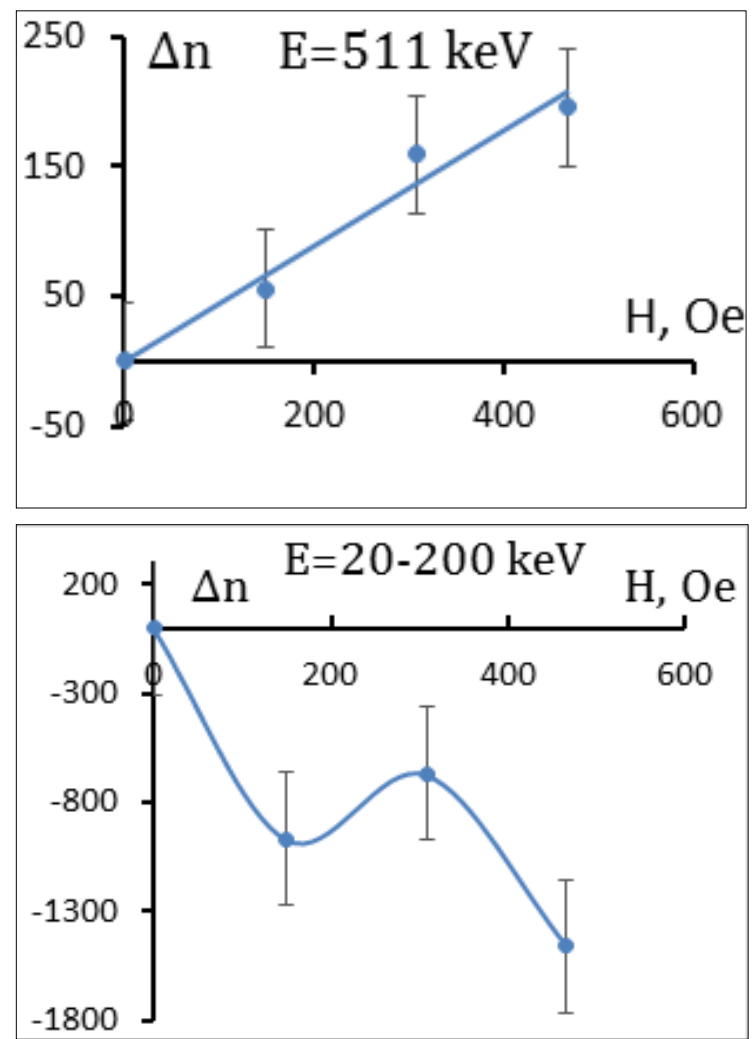

Fig. 5. The dependence of change of the gamma-quanta number $\Delta n$ on the magnetic field $H$.

The number of absorbed gamma-quanta increases in the range of low energies (20-200 keV) with growth in the concentration of nanoparticles $\mathrm{Fe}_{3} \mathrm{O}_{4} / \mathrm{Ta}_{2} \mathrm{O}_{5}$.

\section{Results}

The core-shell nanoparticles $\mathrm{Fe}_{3} \mathrm{O}_{4} / \mathrm{Ta}_{2} \mathrm{O}_{5}$ were studied as a radio-modifier in the X-ray therapy. It is shown that the increase of the concentration of core-shell nanoparticles' leads to the increase of the number of 511 $\mathrm{keV}$ gamma-quanta and the increase of the absorption of the 20-200 keV gamma-rays.

Therefore, the above-described interaction of gamma-ray with nanoparticles $\mathrm{Fe}_{3} \mathrm{O}_{4} / \mathrm{Ta}_{2} \mathrm{O}_{5}$ results in formation of the low-energy secondary gamma quanta which can contribute to disruption of the tumor cells and do not disturb the healthy ones.

The work was supported by financial support in the framework of state task 3.7383.2017/8.9 


\section{References}

1. Hergt, Rudolf, Silvio Dutz, and Michael Röder, Journal of Physics: Condensed Matter 20, 385214 (2008)

2. Moroz, P., S. K. Jones, and B. N. Gray, International Journal of Hyperthermia 18, 267-284 (2002)

3. Gneveckow, U., Jordan, A., Scholz, R., Eckelt, L., Maier-Hauff, K., Johannsen, M., and Wust, P., Biomed. Tech 50, 92-3 (2005)

4. Johannsen, M., Gneveckow, U., Thiesen, B., Taymoorian, K., Cho, C. H., Waldöfner, N., ... and Wust, P., European urology 52, 1653-1662 (2007)

5. Pankhurst, Q. A., Thanh, N. T. K., Jones, S. K., and Dobson, J., Journal of Physics D: Appl. Phys. 42, 224001 (2009)

6. Laurent, S., Dutz, S., Häfeli, U. O., and Mahmoudi, M., Advances in colloid and interface science 166, 8-23 (2011)

7. Murthy, Shashi K., International journal of nanomedicine 2, 129 (2007)

8. Sinibaldi, E., Pensabene, V., Taccola, S., Palagi, S., Menciassi, A., Dario, P., and Mattoli, V., Journal of Nanotechnology in Engineering and Medicine 1, 021008 (2010)

9. Ventrelli, L., Ricotti, L., Menciassi, A., Mazzolai, B., and Mattoli, V., Journal of Nanomaterials 2013, 39 (2013)

10. Alexis, F., Pridgen, E. M., Langer, R., \& Farokhzad, O. C., Drug delivery. Springer Berlin Heidelberg 55-86 (2010)

11. Ghosh Chaudhuri, Rajib, and Santanu Paria, Chemical reviews 112, 2373-2433 (2011)
12. Varadan, Vijay K., Linfeng Chen, and Jining Xie, John Wiley \& Sons (2008)

13. Kurlyandskaya, G. V., Novoselova, I. P., Schupletsova, V. V., Andrade, R., Dunec, N. A., Litvinova, L. S., ... and Khlusov, I. A., Journal of Magnetism and Magnetic Materials 431, 249-254 (2017)

14. Khlusov, I. A., Zagrebin, L. V., Shestov, S. S., Itin, V. I., Sedoi, V. S., Feduschak, T. A., ... and Puchkovskaya, E. S., Bulletin of experimental biology and medicine 145,151-157 (2008)

15. Lin, Hong-Yi, Wei-Yu Chen, and Yu-Chie Chen, Analytical and bioanalytical chemistry 394, 2129-2136 (2009)

16. Novoselova, J. P., Safronov, A. P., Samatov, O. M., Beketov, I. V., Khurshid, H., Nemati, Z., ... and Kurlyandskaya, G. V. IEEE Transactions on Magnetics 50, 1-4 (2014)

17. L. Larionov, M. Zuev, I. Strekalov, S. Sokovnin, Bulletin of the Ural Medical Academy of Science 3, 37 (2011)

18. Bulte, Jeff WM, and Dara L. Kraitchman, NMR in Biomedicine 17, 484-499 (2004)

19. Lei, F., Yan, B., Chen, H. H., Zhang, Q., \&amp; Zhao, J. T., Crystal Growth and Design, 9, 3730-3736 (2009)

20. Steblevskaya N.I., Belobeletskaya M.V., et al. The application for the patent of the Russian Federation №2016132930 from 9.08.2016 (priority). Method of obtaining magnetoactive radiocontrast agent ;

21. I.V. Schegolkov, I.N. Sheyno, V.F. Hohlov, A.A. Lipengolts, Medical physics 4, 12-16 (2010)

22. Kostylev VA Narkevitch BJ "Medical Physics". - M $\therefore$ JSC "Publishing house" Medicine " (2008) 